\title{
Dynamic Spatiotemporal Speed Control of Ultrashort Pulses in Quantum-Dot SOAs
}

\author{
Edeltraud Gehrig, Mike van der Poel, Jesper Mørk, Jørn M. Hvam, and Ortwin Hess
}

\begin{abstract}
We present theoretical and experimental results on the propagation of ultrashort pulses in quantum-dot (QD) laser amplifiers. The propagation time of the light pulses is controlled by the pulse itself (self-induced speed control) or by injection of a second pump pulse (external speed control). Our simulations on the basis of spatially and temporally resolved QD Maxwell-Bloch equations reveal that the excitation and relaxation dynamics induced by the propagating pulse or a pump pulse within the active charge carrier system leads to a complex gain and index dynamics that may either speed up or slow down the propagating light pulse. The physical effects allowing for the dynamic speed control could be ascribed to complex (coherent and incoherent) level dynamics leading to dynamic gain saturation and index dispersion. The dependence of the propagation time on injection current density and pulse energy is discussed. The numerical results of pulse reshaping and propagation times in the gain and absorptions regime are compared to experimental results.
\end{abstract}

Index Terms-Quantum dots (QDs), semiconductor optical amplifiers, ultrafast optics.

\section{INTRODUCTION}

$\mathbf{T}$ UNABLE pulse propagation time in optical media by controllable modification of the nonlinear index of refraction has in recent years caused great excitement as it opens the possibility of a new class of optical components with optical memory capability. First experiments by Hau et al. [1] used the technique of electromagnetically induced transparency to slow down light propagation velocity to $17 \mathrm{~m} / \mathrm{s}$. More recently, Bigelow et al. demonstrated light slowdown to $59 \mathrm{~m} / \mathrm{s}$ [2] by the technique of coherent population oscillation (CPO). One advantage of $\mathrm{CPO}$ compared to other slow-light techniques is its insensitivity to the quantum mechanical coherence of the optical dipoles in the optical medium interacting with the signal light. Thus, the above mentioned result using $\mathrm{CPO}$ could be realized at room temperature. Very recent results demonstrating CPO in room temperature semiconductor components such as a quantum well saturable absorber [3] and a quantum-dot (QD) optical amplifier [4] suggest that tunable slow light may be realized in practical components.

A fundamental issue in slow light schemes is to achieve a large slowdown over a sufficiently large bandwidth. The very large slowdown in [2], for instance, was at the cost of a very

Manuscript received March 29, 2006; revised June 22, 2006. This work was supported in part by the EU-IST project DOTCOM under Project No. IST-200028713.

E. Gehrig and O. Hess are with the Advanced Technology Institute, School of Electronics and Physical Sciences, University of Surrey, GU2 7XH Surrey, U.K. (e-mail: e.gehrig@surrey.ac.uk; o.hess@ surrey.ac.uk).

M. van der Poel, J. Mørk, and J. M. Hvam are with the Communication, Optics and Materials (COM) Research Center, Technical University of Denmark, DK-2800 Lyngby, Denmark (e-mail:mvp@com.dtu.dk; jm@com.dtu.dk; hvam@com.dtu.dk).

Digital Object Identifier 10.1109/JQE.2006.881632

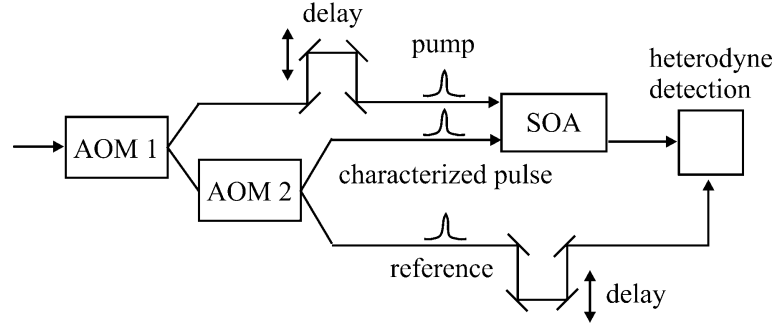

Fig. 1. Schematic overview of the experimental setup.

small bandwidth of $36 \mathrm{~Hz}$. In [3] and [4], the slow down were more modest, corresponding to a group index change of $\Delta n_{g}=$ 7 and $\Delta n_{g}=4 \cdot 10^{-3}$, respectively. This, however, was achieved at correspondingly larger bandwidths of $20 \mathrm{GHz}$ and $2.6 \mathrm{THz}$, respectively.

In this paper, we numerically analyse the light induced delay and advancement of an ultrashort optical pulse propagating in a QD SOA corresponding to the results reported in [4]. Due to the specific physical properties of the active medium (e.g., discrete energy levels, high gain, low threshold current, low alpha factor [5] and high-speed modulation [6]) QD based semiconductor optical amplifiers (SOAs) represent promising systems for novel optoelectronic components in data storage and telecommunication. The simulations are based on spatially resolved QD Maxwell-Bloch equations [7] that take into account spatiotemporal light propagation in the waveguiding structure, the spatialdependence and microscopic coupling between light and carriers as well as dynamic excitation and relaxation of the charge carrier plasma via carrier-carrier and carrier-phonon scattering. Our numerical results provide a spatiotemporally resolved analysis of gain and index dynamics responsible for the dynamic shaping of the propagating light pulse. The theoretical results are compared to an experimental investigation performed with a pump-probe setup with heterodyne detection.

\section{EXPERIMENT AND SAMPLE DETAILS}

Cross-correlation traces of a pulse propagated through a QD SOA and a reference pulse travelling outside the device were recorded using a heterodyne detection technique [8] (Fig. 1). From the temporal profile of the characterized pulse, changes in the pulse propagation time and shape were recorded when parameters such as pulse energy and SOA bias current were changed. In some of the experiments an additional pump pulse was launched into the SOA at a specific time before the characterized pulse, to measure the corresponding pump-induced changes in the characterized pulse. The characterized pulse (probe pulse) and reference pulse were derived from a single 
pulse from a laser source by means of two acoustooptic modulators (AOMs) operating at different frequencies. Changing the drive power of AOM 2 allowed the intensity of the characterized pulse to be changed by two orders of magnitude from the small signal regime to saturation without changing the relative timing of the pulses. In the experiments where a pump-pulse was launched into the SOA, the pump pulse energy was in the saturation regime, while the characterized pulse was at a much lower energy and in the small-signal regime.

The component under investigation was a ridge-waveguide QD SOA of length $2 \mathrm{~mm}$ and width $7 \mu \mathrm{m}$. The active layer consisted of five sheets of self-organized InAs dots grown on GaAs and overgrown with a 5-nm InGaAs layer [9]. Each layer had a dot surface density of $4 \cdot 10^{10} \mathrm{~cm}^{-2}$ and the dot ensemble displayed a ground state emission wavelength centered at $1260 \mathrm{~nm}$ and with an inhomogeneous linewidth of $90 \mathrm{~nm}$. The ultrafast gain and index dynamics of this SOA was characterized experimentally and modelled theoretically with a phenomenological model in [10]. The laser source delivering the short pulses was a Ti:sapphire oscillator, regenerative amplifier, and optical parametric amplifier in cascade. The pulses were coupled in and out of the waveguide by means of microscope objectives. Before each measurement run alignment of the microscope objectives was optimized for maximum coupling to the waveguide. We find the coupling loss to be about $10 \mathrm{~dB}$ at each facet. We also find a day-to-day variation in the coupling loss so there is a variation of the pulse energy coupled into the waveguide for a given pulse energy measured in free-space before incoupling. For this reason, it is necessary to allow a pulse energy variation in the order of a factor 2 in the modelling of the experiment.

\section{CARRIER DYNAMICS AND SPATIOTEMPORAL GAIN AND INDEX DYNAMICS}

Our computational modelling of pulse propagation in QD lasers is based on a mesoscopic theoretical description [7] (see appendix). The propagation of an optical light pulse (in the model realized via the boundary conditions of the light fields at the facets) induces highly nonequilibrium distributions in the charge carrier ensemble populating the dots. The dots of our considered system have a pyramidal shape, with typical base length of $14 \mathrm{~nm}$ leading to three electron level energies (180, 100 , and $85 \mathrm{meV}$, relative to the band edge of the conduction band in the wetting layer) and five hole level energies (110, 90, 75,70 , and $55 \mathrm{meV}$, relative to the band edge of the valence band in the wetting layer). As an example, Fig. 2 visualizes the carrier dynamics in the three electron levels (e1-e3) and five hole levels (h1-h5) during the propagation of a resonant light pulse. At the start of the calculation the injection current density has been set to $2 \cdot I_{\mathrm{thr}}$. The input energy of the pulse is $E_{\mathrm{in}}=0.8 \cdot E_{s}$ where $E_{s}$ is the pulse saturation energy (defined as the energy at which the gain has decreased by $3 \mathrm{~dB}$ [17]).

Depending on the individual dipole matrix elements and level structure a dynamic depletion of the level occurs. The specific shape of this dynamic level-hole burning thereby depends on injection current density, input pulse energy and intradot scattering. In the given example, the level separation is near the longitudinal optical (LO) phonon energy leading to a dynamic exchange of carriers on the individual levels via emission and
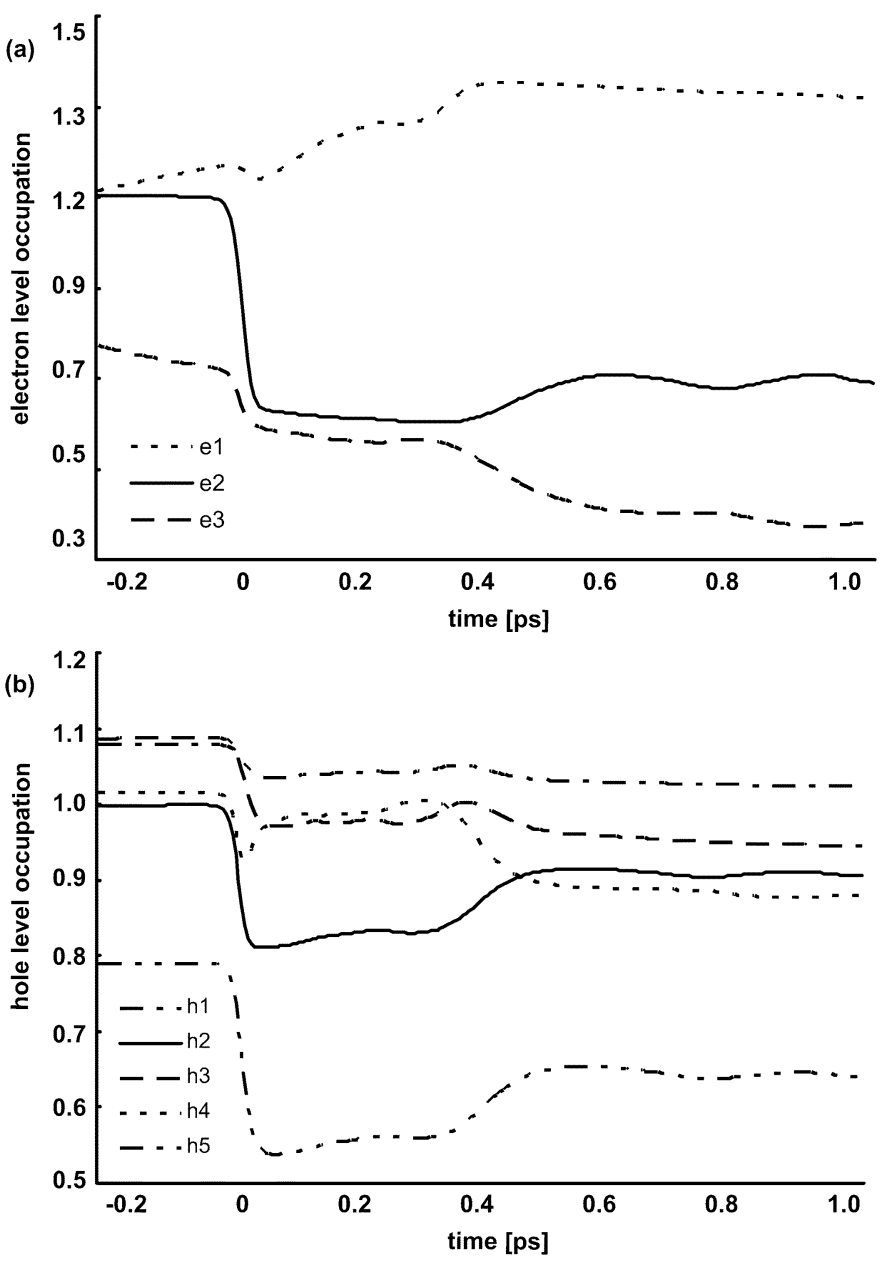

Fig. 2. Dynamics of level occupations in the(a) electron and (b) hole levels calculated at the center of the output facet during the passage of the pulse.

absorption of LO phonons. In particular, the refilling of the resonant dot levels from higher-energy levels (e.g., e3, h5) may lead to a partial recovery of the gain [18].

The changes in the carrier ensemble are directly correlated to corresponding changes in the dipole dynamics coupling the individual electron and holes levels. The sum (over all electron hole level combinations) of these microscopic dipole densities composes the polarization entering the wave equation. Dividing the real and imaginary part of the polarization $(p)$ by the optical fields $(E)$ leads to the complex susceptibility which directly revals the index and gain dynamics via its real and imaginary part, respectively. This definition is in accordance with the definition of the susceptiblity $(\chi)$ via the expression $p=\chi \cdot E$ that is typically used in phenomenological modelling (after elimination of the polarization dynamics). We would like to point out, however, that the complex dynamics of the polarization is fully taken into account in the simulations via the Bloch equations [7].

As a first example we will consider the absorption regime and analyze the dynamics of the susectibility in a dot ensemble where the carrier distribution is below the transparency level: Figs. 3 and 4 show the dynamics of the real (Fig. 3) and imaginary part (Fig. 4) of the susceptibility at the output facet of the amplifier during the passage of a light pulse. Shown are the dynamic distributions in a time window of $1.5 \mathrm{ps}$. The zero 
(a)

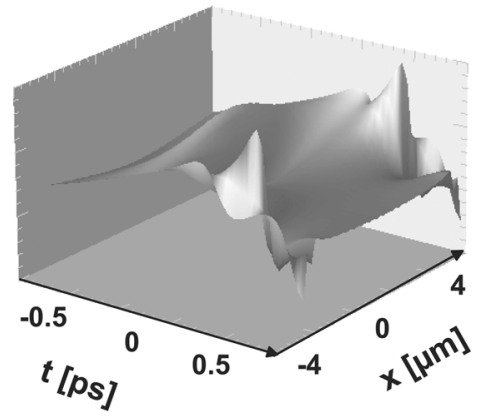

(b)

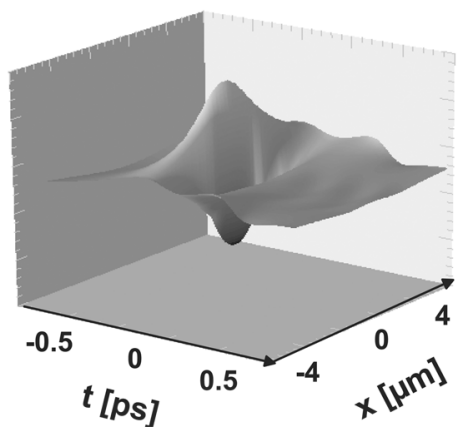

Fig. 3. Index dynamics in the absorption regime. The distributions were calculated at the output facet of the QD laser for an input pulse energy of (a) $E_{\text {in }}=$ $0.1 E_{s}$ and (b) $E_{\text {in }}=1 E_{s}$.

(a)

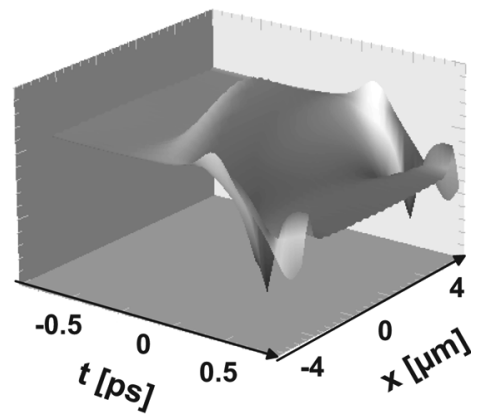

(b)

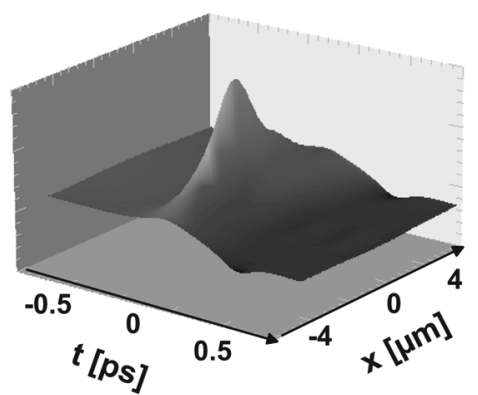

Fig. 4. Gain dynamics in the absorption regime: The distributions were calculated at the output facet of the QD laser for an input pulse energy of (a) $E_{\text {in }}=$ $0.1 E_{s}$ and (b) $E_{\text {in }}=1 E_{s}$.

marks the pulse center. The input energy of the pulse is $E_{\mathrm{in}}=$ $0.1 E_{s}$ (a) and $E_{\text {in }}=1 E_{s}$ (b). The spatial shape of the distributions thereby directly reflects the intradot dynamics: The injected light pulse leads to a carrier accumulation. In combination with the complex relaxation dynamics following the excitation of the charge carriers this leads to a characteristic shape of the induced index and gain. The real part (revealing the dynamics of the index) shows a distorted dispersion shape, the imaginary (a)

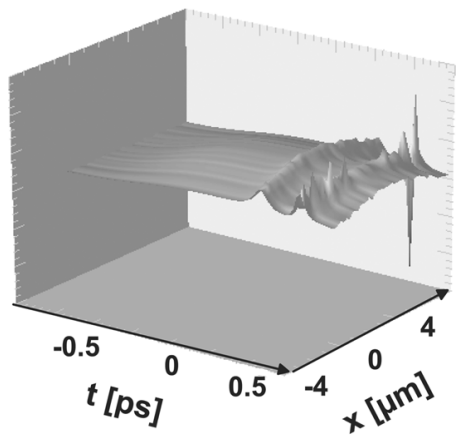

(b)

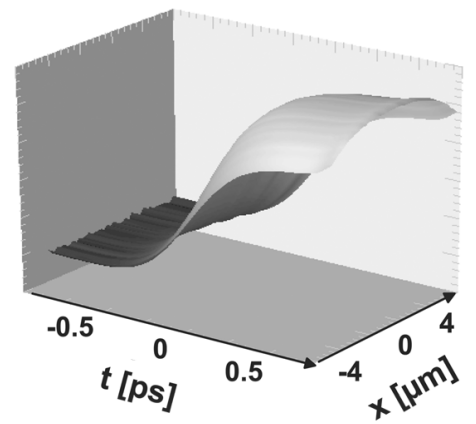

Fig. 5. Index dynamics in the amplification regime: The distributions were calculated at the output facet of the QD laser for an input pulse energy of (a) $E_{\text {in }}=$ $0.1 E_{s}$ and (b) $E_{\text {in }}=1 E_{s}$.

(a)

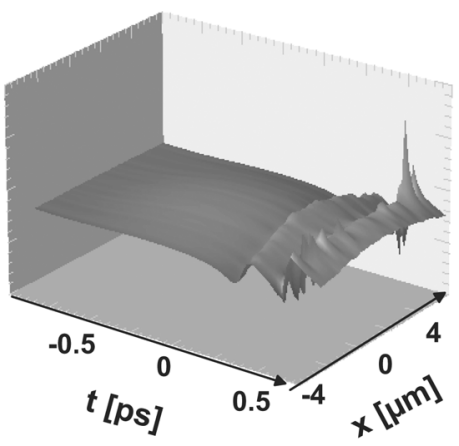

(b)

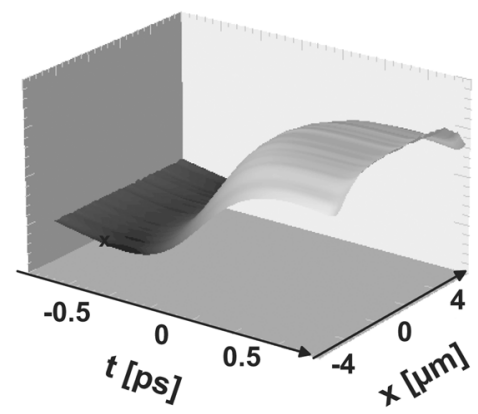

Fig. 6. Gain dynamics in the amplification regime: The distributions were calculated at the output facet of the QD laser for an input pulse energy of (a) $E_{\text {in }}=$ $0.1 E_{s}$ and (b) $E_{\text {in }}=1 E_{s}$

part (revealing gain dynamics) visualizes the absorption (negative values) and a partial amplification (positive values) in the case of high input energies (generated by the pulse-induced carrier accumulation). In the gain regime (Figs. 5 and 6 ) the injected light pulse leads to a carrier depletion in the inverted medium and the index dispersion changes its sign. For low intput powers the depletion of the ground state levels and partial refilling via 


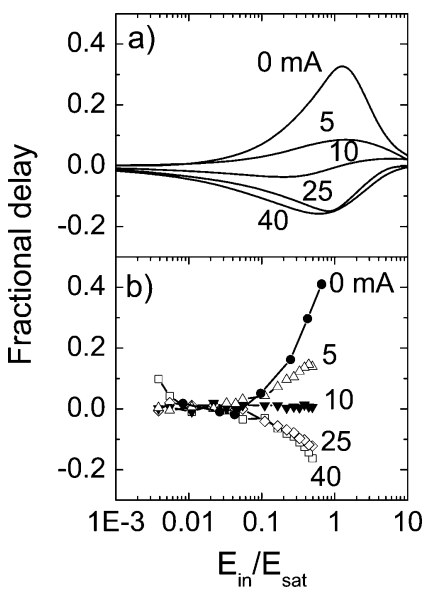

Fig. 7. Relative pulse shift in dependence on input pulse energy for different currents. (a) Results of the numerical modelling. (b) Experimental results.

intradot scattering (from higher energy states) and carrier capture from the wetting layer states lead to characteristic modulations, i.e., scattering effects dominate the behaviour (leading to singularities). For high input pulse energy the propagating light pulse induces a strong level hole-burning leading to a a characteristic rise in the spatial index and gain [Figs. 5(b) and 6(b)]. As a consequence the carrier dynamics is not entirely relaxed after the passage of the pulse and the duration of the gain and index changes significantly exceeds the duration of the pulse.

The observed effects can be explained and summarized as follows.

1) The complex carrier dynamics that is typical for spatially inhomogeneous QD ensemble leads to dynamic changes in gain and index that do not necessarily display the typical resonance-like behavior but generally show a complex gain dynamics and distorted index dispersion. This originates from the difference in the timescales on which the physical processes take place: Ultrafast (fs.ps) carrier and carrierphonon scattering processes within the dots, propagation of the light pulse (ps regime) and slow re-establishment of the spatial inversion due to the injection current (ns regime).

2) The observed gain and index dynamics is a consequence of the dynamic space-dependent light-matter interactions that take place during the propagation.

3) The spatiotemporally varying gain and index reveal the reasons for changes in the propagation times: Reshaping of the gain (i.e., saturation) induces a shift of the pulse center leading to a propagation time that will depend on current and power. Reshaping of the index leads to velocity changes and to the generation of new frequencies within the pulse shape. Both effects affect the propagation time.

It is this complex interplay of index dispersion and gain dynamics that is responsible for the passage time (dynamic acceleration and slow-down) and dynamic shaping of the propagating light pulse which will be discussed in the next section.

\section{SElf-Induced PRopagation CONTROL}

In a next step, we systematically vary the input pulse energy and injection current and calculate the pulse shift. The results of the simulations are summarized in Fig. 7(a) and com-

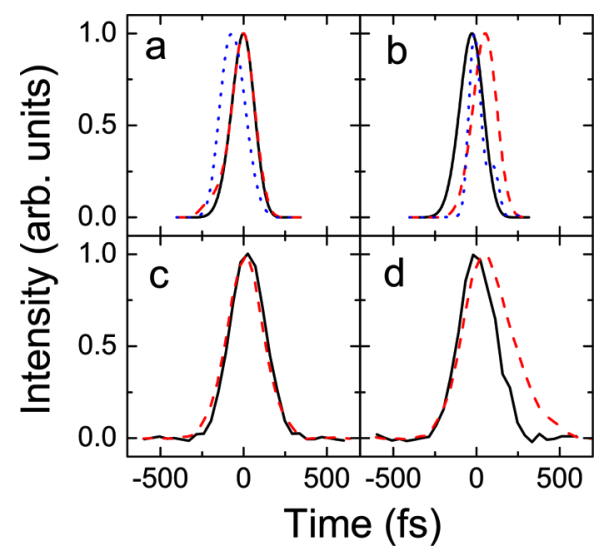

Fig. 8. Calculated and experimental pulse shapes after propagation in the QD SOA. (a)-(b) Calculated pulse shapes for the amplification and absorption regime, respectively. (c)-(d) Experimental cross-correlation traces of the pulse after propagation in the SOA with a $170 \mathrm{fs}$ (FWHM) Gaussian pulse travelling outside the laser. Note that the experimental traces are wider than the theoretical ones because they are convolutions of the SOA pulse and an external one.

pared to earlier reported [4] experimental results obtained with the pump-probe setup Fig. 7(b). The theoretical results are in good agreement with the experiment. The pulse shift is considered relative to its position in the unsaturated device and calculated relative to the pulse duration (180 fs). Please note that a logarithmic scale has been chosen for the input energy. In the amplification regime (i.e., at injection currents larger than the transparency current of $15 \mathrm{~mA}$ ) a gradual relative pulse acceleration of up to $20 \%$ (corresponding to an absolute value of $36 \mathrm{fs}$ ) is reached with increasing pulse energy. Near saturation, the leading edge of the propagating light pulse induces a dynamic level hole-burning in the charge carrier system leading to a reduction of the gain available for the trailing part of the pulse. In combination with the pulse-induced index dispersion this leads to a pulse acceleration, i.e., a positive pulse shift. For pulse energies significantly beyond the saturation energy the pulse shift decreases. In this regime the level occupation is already entirely depleted by the very front part of the pulse. In the absorption regime the sign of the relative pulse shift changes and relative pulse shifts of up to $40 \%$ (corresponding to an absolute value of $72 \mathrm{fs}$ ) are obtained. In this case an increase in pulse energy leads to a bleaching of the absorption by the rising edge of the pulse leading to a reduced absorption for the trailing pulse part. The maximum pulse shift is larger than in the amplification regime. This originates from the partial gain recovery due to intradot relaxation that is typical for QD lasers below transparency [10]. In [4] it was shown how salient features of the observed self-slowdown or speed up can be explained with a gain saturation model. Here, we demonstrate (using a spatially and temporally resolved Maxwell-Bloch approach) that it is the (spatially varying) interplay of both, coherent gain and index dynamics as well as incoherent scattering that affects the propagation time and shape of a light pulse passing a QD laser amplifier.

\section{Dynamic Pulse Shaping}

The dynamics of gain and index discussed in Section IV is not only responsible for a dynamic acceleration or slow-down of the pulse but also affects its shape. Fig. 8 summarizes (a)-(b) cal- 
culated and (c)-(d) measured [4] pulse shapes in the (a), (c) amplification regime and (b), (d) absorption regime, respectively. Shown are results for input pulse energies in the small signal regime (solid line), near saturation (dashed line) and beyond the saturation regime (dotted line). In the amplification regime, a typical temporal asymmetry can be seen near saturation, in addition to the temporal shift of the pulse center that has already been discussed in the previous section. Similarly, the complex gain and index dynamics lead to corresponding changes in the pulse shape in the absorption regime. The absolute value of the temporal shift of the pulse center in both the amplification and the absorption regime, is strongly influenced by the depletion and generation of carriers by the propagating light pulse. However, an additional influence due to incoherent scattering processes such as, for example, intradot carrier relaxation via emission and absorption of phonons occurs. Basically, these effects lead to two changes in the spatiotemporal dynamics during pulse propagation: First, the dynamic exchange of carriers between different levels via emission and absorption of phonons leads to a rather uniform depletion of all levels whereas a more selective depletion of individual levels (with highest dipole matrix elements and level energies in resonance with the pulse energy) would occur in a decoupled system [10]. As a consequence, saturation in a dot system with decoupled levels is already reached for smaller pulse energies. Secondly, the complex scattering dynamics is - via the QD Bloch equations-directly related to corresponding changes in the dipole dynamics entering the wave equations. As a result, changes in the level occupations are directly transferred to the light field dynamics. The coupling of spectral with spatiotemporal degrees of freedom realized by the mutual interplay of (intradot and dot-to-wetting layer) scattering, light propagation, diffusion and spatiotemporal gain and index dynamics then leads to a dynamic reshaping of the pulse. This is particularly pronounced in the ultrashort time regime and near transparency where a comparable number of filled and empty states coexist leading to a strong level dynamics.

In order to visualize the influence of dynamic scattering processes we have calculated the pulse shape in the absorption regime $\left(E=0.5 E_{s}\right)$ at the output facet of our QD laser and compared it with the pulse shape evolution in a QD laser device with decoupled dot levels, i.e., where the dot levels are separated by more than the LO phonon energy so that emission and absorption of phonons is negligible. The resulting pulse shape of the decoupled system, displayed in Fig. 9(a), is characterized by a comparatively smooth pulse envelope. In contrast, the shape of a light pulse after the passage in the dot ensemble with coupled energy levels Fig. 9(b) shows a completely different behavior: In this system, the transfer of carrier scattering processes to the propagating light field dynamics induces a complex reshaping of the light pulses. This is particularly pronounced in the trailing part of the light pulse where a complex carrier relaxation occurs in response to the pulse excitation.

\section{Pump-Probe Propagation Control}

The dynamic shaping of gain and index distributions induced by a propagating light pulse directly leads to the idea of a pumpprobe set-up: In this configuration the injection of a high-energy pump pulse should allow the control of the propagation time of
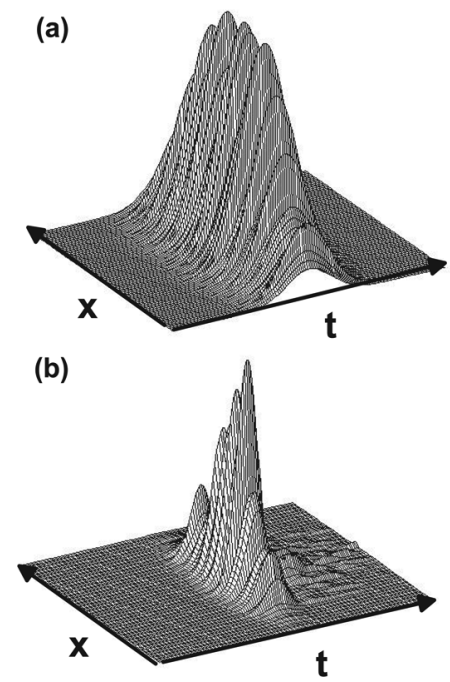

Fig. 9. Spatially resolved pulse shapes at the output facet $\left(E=0.5 E_{s}\right)$ in a QD laser with (a) decoupled dot levels and in a QD laser with energy levels that coupled by (b) dynamic carrier-phonon relaxation processes.

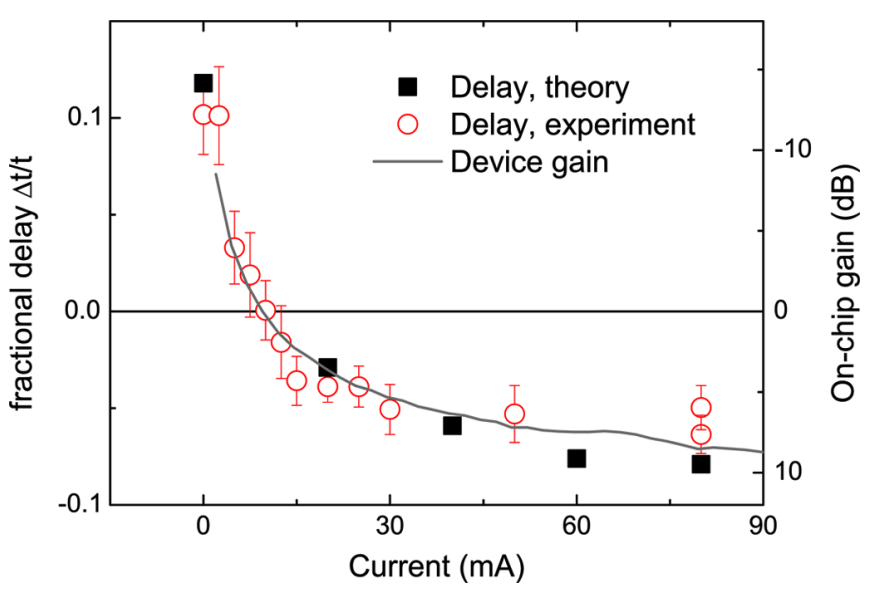

Fig. 10. Left axis: pump-induced relative delay of the probe pulse in dependence on injection current for zero pump-probe delay. Filled and open symbols show calculated and measured results, respectively. Right axis: independently measured small signal gain of pulse.

the probe pulse. In a next step we thus consider the propagation of a pulse in a QD ensemble that has been modified by a preceding pump pulse. The energy of the pump pulse is near the saturation energy of the amplifier, the input energy of the probe pulse lies in the small signal regime. The duration of the two pulses is $170 \mathrm{fs}$ and their wavelength is $1273 \mathrm{~nm}$. During the propagation in the QD medium the probe pulse interacts with a carrier distribution that has been excited by the pump pulse. By varying the delay between the two pulses or the injection current in the QD laser it should thus be possible to tune the delay of the probe pulse. Fig. 10 shows the calculated and measured pump-induced delay of the probe pulse in dependence on injection current for a zero delay between pump and probe pulse. The theory is in good agreement with the experimentally obtained results: At $0 \mathrm{~mA}$ a maximum relative delay of $12 \%$ is obtained corresponding to an absolute delay of $20 \mathrm{fs}$. With increasing current the delay is reduced and changes its sign. The 


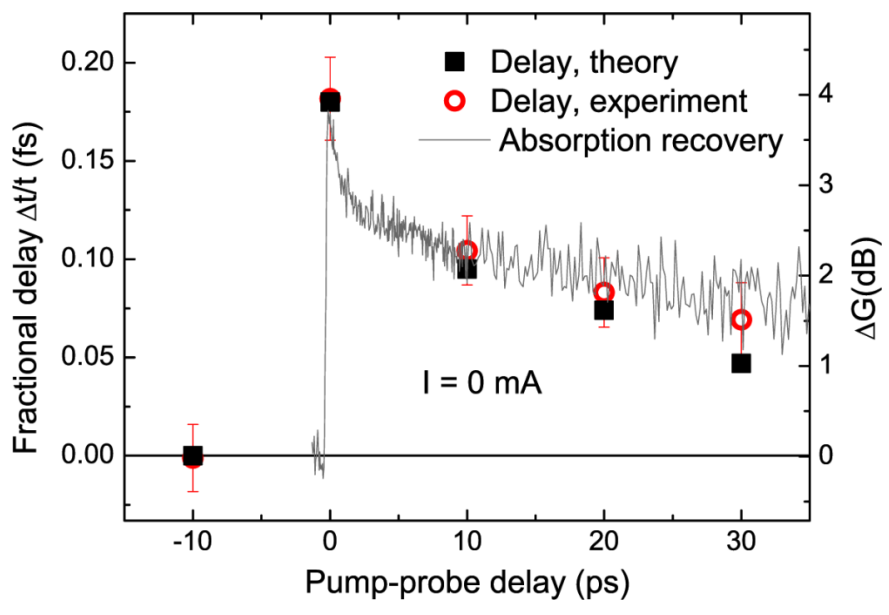

Fig. 11. Left axis: pump-induced relative delay of the probe pulse in dependence on delay between pump and probe pulse at $0 \mathrm{~mA}$. Filled and open symbols show calculated and measured results, respectively. Right axis: independently measured differential gain of a weak pulse following a strong pulse.

results can be interpreted in analogy to the results obtained for the single-pulse configuration: The excited carrier system induced by the pump pulse leads to corresponding gain and index distributions (see also Figs. 3-6). For low injection currents the probe pulse interacts with almost empty dots leading to carrier accumulation. The resulting gain dynamics and index dispersion then results in a delay of the probe pulse. With increasing injection current the dots of the amplifier are significantly filled before the passage of the pulses. As a consequence, a dynamic level hole-burning arises leading to a speed up of the propagating probe pulse. For a given value of the probe energy the injection current consequently can be used to tune the relative shift experienced by the probe pulse. A second control parameter is given by the pump-probe delay: After their excitation the carriers relax via intradot scattering and carrier capture towards their quasi-equilibrium distributions. This relaxation dynamics typically occurs on a picosecond time scale. The gain and index dynamics and the resulting delay experienced by the probe pulse consequently depend on the time between the carrier excitation (induced by the pump pulse) and the passage of the probe pulse. Fig. 11 summarizes the numerical and experimental results on the dependence of the relative pulse shift on pump-probe delay. The injection current has been set to zero. The measured dependence of the pulse shift on the pump-probe delay is qualitatively confirmed by the theoretical curve. The reason for the slightly faster decay seen in the simulated results is associated to uncertainties in some of the material parameters such as, e.g., disorder in the dot distribution. If the pump pulse is behind the probe pulse the probe pulse does not experience the modifications in the carrier system generated by the probe pulse and the relative pulse delay equals zero. At zero delay between pump and probe pulse a maximum relative delay of $12 \%$ of the probe pulse (corresponding to an absolute value of $34 \mathrm{fs}$ ) is obtained. With increasing pump-probe delay the probe pulse experiences the gain dynamics and index dispersion of the partially relaxed carrier system leading to a characteristic decrease of the relative pulse shift.

\section{CONCLUSION}

We have investigated the propagation time of ultrashort light pulses in QD semiconductor optical amplifiers. A theoretical analysis on the basis of spatiotemporally resolved QD Maxwell-Bloch equations revealed a complex interplay of gain and index dynamics that affect the propagation time and dynamically shape the light pulse during its propagation. It could be demonstrated that the delay of the emitted light pulse strongly depends on injection current and input pulse energy. In the amplification regime, an acceleration of the pulse of up to $20 \%$ of its duration has been obtained for sufficiently high input pulse energy values whereas a significant slow down of $40 \%$ could be observed in absorption. The computational results was in good agreement with experimental pump-probe measurements. The theoretical analysis revealed that it is the interplay of both, coherent light-matter coupling and incoherent scattering processes such as carrier capture and intradot carrier-phonon interactions that affect the ultrashort carrier dynamics and consequently the gain and index dynamics in the amplifier. As a result, the propagation time and, in particular, the shape of a light pulse after its passage in the QD amplifier are determined by spatiospectral gain saturation, index dynamics and ultrafast carrier scattering. Our computational and experimental results clearly indicate the potential of QD semiconductor optical amplifiers for a speed control of ultrashort high-bandwidth light pulses. Such functionality can provide fine-tuning of pulse time position which is important in e.g., optical time domain multiplexing (OTDM) systems.

\section{APPENDIX \\ QD MAXWELL-BLOCH EQUATIONS}

The spatially resolved QD Maxwell-Bloch equations describe the spatiotemporal light field and inter/intralevel carrier dynamics in each dot of a typical QD ensemble in QD lasers. Starting from the quantum kinetic equations of motion of the single particle density matrices with respect to the carrierphonon Hamiltonian, factorizing the intraband and interlevel matrices into single-particle density matrices and using the Markov approximation (i.e., assuming slowly varying distributions) one obtains the following equations for the dynamics of the occupation of electrons ( $e$, level index $i$ ) and holes ( $h$ level index $j), n^{\mathrm{e}, \mathrm{h}}$, and the dynamics of the interlevel polarizations $p^{ \pm}$(coupled to the forward $(+)$ and backward $(-)$ propagating optical fields)

$$
\begin{aligned}
\frac{\partial n_{i, j}^{e, h}}{\partial t}= & \Lambda^{e, h}+g_{i, j}^{e, h}-\gamma_{\mathrm{nr}} n_{i, j}^{e, h}-\sum_{j, i} \Gamma_{\mathrm{sp}} n_{i, j}^{e, h} \cdot n_{j, i}^{h, e} \\
& +\left.\frac{\partial n_{i, j}^{e, h}}{\partial t}\right|_{\mathrm{QD}} ^{\mathrm{p}-\mathrm{ph}}+\left.\frac{\partial n_{i, j}^{e, h}}{\partial t}\right|_{\mathrm{QD}-\mathrm{WL}} \\
\frac{\partial p_{j i}^{ \pm}}{\partial t}= & -\left(i \bar{\omega}_{j i}+\gamma_{\mathrm{p}}\right) p_{j i}^{ \pm}-\frac{i}{\hbar}\left[n_{i}^{e}+n_{j}^{h}\right] \mathcal{U}^{ \pm} \\
& -\frac{i}{\hbar} \delta \mathcal{U}_{\mathrm{nl}}^{ \pm}+\left.\frac{\partial p_{j i}^{ \pm}}{\partial t}\right|_{\mathrm{QD}} ^{\mathrm{p}-\mathrm{ph}} .
\end{aligned}
$$

$\gamma_{\mathrm{nr}}$ represents the rate due to nonradiative recombination and $\gamma_{p}$ denotes the dephasing rate of the interlevel dipole. The pump 
term $\Lambda^{e, h}$ mesoscopically represents the carrier injection and includes the pump-blocking effect. The generation rate $g_{i, j}^{e, h}=$ $\operatorname{Re}\left[(i) /(\hbar) \sum_{j, i}\left[\left(\mathcal{U}^{+} p_{j, i}^{+*}+\mathcal{U}^{-} p_{j, i}^{-*}\right)-\left(\mathcal{U}^{+*} p_{j, i}^{+}+\mathcal{U}^{-*} p_{j, i}^{-}\right)\right]\right]$ depends on the interlevel polarization $p$ and on the optical field contributions of spontaneous and induced emission constituting the local field $\mathcal{U}^{ \pm}$. The local fields $\mathcal{U}^{ \pm}=\vec{d}_{j i} \vec{E}^{ \pm}+\delta \mathcal{U}^{ \pm}$are composed of the optical light field contributions $E^{ \pm}$and those induced by Coulomb screening in each QD and by the Coulomb interactions between the carriers in the QD and the carriers in the wetting layer, $\delta \mathcal{U} . \vec{d}_{j i}$ is the interlevel dipole matrix element. The interlevel polarization depends via $\bar{\omega}_{j i}=\hbar^{-1}\left(\mathcal{E}^{e}+\mathcal{E}^{h}\right)-\omega$ ( $\omega$ is the frequency of the propagating light fields) on the carrier energies. The last terms in (1) describe scattering processes such as e.g., emission and absorption of phonons (approximated by their quasi-equilibrium distribution) and carrier scattering to wetting layer that are dynamically included on a mesoscopic level. The dynamics of the carriers in the surrounding wetting layer of the dots is described with a diffusion equation [7]. The material properties of the QD ensemble (dipole matrix elements and energy levels) are considered via effective parameters [16]. Spatial fluctuations in material properties (dot size, level energies, dot distribution) are taken into account via a space dependent parameter set. Via the generation rate and the dipole dynamics at each location in the laser structure the carrier dynamics is fundamentally linked to the light field dynamics that is described by the wave equation

$$
\begin{aligned}
\frac{\partial^{2}}{\partial x^{2}} \vec{E}^{ \pm} \pm 2 i \beta \frac{\partial}{\partial z} \vec{E}^{ \pm}+\frac{\partial^{2}}{\partial z^{2}} \vec{E}^{ \pm}+\frac{2 i \omega}{c^{2}} \frac{\partial}{\partial t} \vec{E}^{ \pm}-\frac{1}{c^{2}} \frac{\partial^{2}}{\partial t^{2}} \vec{E}^{ \pm} \\
=-\mu_{0} \omega^{2} \vec{P}^{ \pm}-2 i \omega \frac{\partial}{\partial t} \vec{P}^{ \pm}
\end{aligned}
$$

where the polarization of the active semiconductor medium, $\vec{P}^{ \pm}=\sum_{i, j} \vec{d}_{i j} p_{j i}^{ \pm}$is the source of the optical fields. At every grid point and for each time step, the physical quantities are calculated on the basis of finite difference methods (on a numerical grid with regular mesh-size) using the Hopscotch [19] method as a general scheme. Typical grid sizes are a few microns, the step for the integration in time is in the regime $0.5-1 \mathrm{fs}$.

\section{ACKNOWLEDGMENT}

The authors would like to thank A. Kovsh, Ioffe Institute (now at NL Semiconductor) for growing the QD sample. M. Laemmlin and D. Bimberg of Technische Universität Berlin are acknowledged for wafer processing.

\section{REFERENCES}

[1] L. V. Hau, S. E. Harris, Z. Dutton, and C. H. Behroozi, Nature, vol. 397, pp. 594-598, 1999.

[2] M. S. Bigelow, N. N. Lepeshkin, and R. W. Boyd, Phys. Rev. Lett., vol. 90, p. 113903, 2003.

[3] J. Mørk, R. Kjær, M. van der Poel, and K. Yvind, Opt. Exp., vol. 13, pp. 8136-8145, 2005.

[4] M. van der Poel, J. Mørk, and J. M. Hvam, Opt. Exp., vol. 13, pp. 8032-8037, 2005.

[5] S. Ghosh, S. Pradhan, and P. Bhattacharya, Appl. Phys. Lett., vol. 81, pp. 3055-3057, 2002.

[6] N. Hatori, K. Otsubo, M. Ishida, T. Akiyama, Y. Nakata, H. Ebe, S. Okumura, T. Yamamoto, M. Sugawara, and Y. Arakawa, " $20^{\circ} \mathrm{C}-70^{\circ} \mathrm{C}$ temperature independent $10 \mathrm{~Gb} / \mathrm{s}$ operation of a directly modulated laser diode using p-doped quantum dots," presented at the 30th European Conf. Opt. Commun., 2004, paper Th4.3.4.
[7] E. Gehrig and O. Hess, Phys. Rev. A, vol. 65, p. 033804, 2002.

[8] P. Borri, W. Langbein, J. Mørk, and J. M. Hvam, Opt. Comm., vol. 169, p. 317, 1999.

[9] A. R. Kovsh, J. Cryst. Growth, vol. 251, pp. 729-736, 2003.

[10] M. van der Poel, E. Gehrig, O. Hess, D. Birkedal, and J. M. Hvam, IEEE J. Quantum Electron., vol. 41, no. 9, pp. 1115-1123, Sep. 2005.

[11] J. Schilp, T. Kuhn, and G. Mahler, Phys. Rev. B., vol. 50, pp. 5435-5447, 1994.

[12] T. Inoshita and H. Sakaki, Phys. Rev. B, vol. 46, pp. 7260-7263, 1992.

[13] A. V. Uskov, J. McInnerney, F. Adler, H. Schweizer, and M. H. Pilkuhn, Appl. Phys. Lett., vol. 72, pp. 58-60, 1998.

[14] J. L. Pan and P. L. Hagelstein, Phys. Rev. B, vol. 49, pp. 2554-2575, 1994.

[15] A. V. Uskov, K. Nishi, and R. Lang, Appl. Phys. Lett., vol. 74, pp. 3081-3083, 1999.

[16] O. Stier, M. Grundmann, and D. Bimberg, Phys. Rev. B, vol. 59, pp. 5688-5701, 1999.

[17] T. Berg and J. Mørk, IEEE J. Quantum Electron., vol. 40, no. 11, pp. 1527-1529, Nov. 2004.

[18] T. W. Berg, S. Bischoff, I. Magnusdottir, and J. Mørk, IEEE Photon. Technol. Lett., vol. 13, no. 6, pp. 541-543, Jun. 2001.

[19] I. S. Greig and J. D. Morris, J. Comp. Phys., vol. 20, pp. 64-80, 1976.

Edeltraud Gehrig received the diploma and Dr.rer.nat degrees in physics from the University of Kaiserslautern, Kaiserslautern, Germany, in 1995 and 1999, respectively.

From 2000 to 2003, she was a Senior Research Associate with the Theoretical Quantum Electronics Group, DLR Stuttgart, Stuttgart, Germany. Since April 2003, she has been a Senior Research Associate with the University of Surrey, Surrey, U.K. Her research includes biophotonic and biodynamic systems, molecular motors, ultrafast nonlinear dynamics, dynamics and control of high-power semiconductor lasers and amplifiers, luminescence, and quantum-dot lasers.

Mike van der Poel received the M.Sc. degree in physics and the Ph.D. degree from the University of Copenhagen, Copenhagen, Denmark, in 1997 and 2001, respectively. His dissertation was on laser cooling and atomic collisions.

Since 2001, he has been an Assistant Professor at the Communication, Optics and Materials (COM) Research Center, Technical University of Denmark, Lyngby, Denmark. His research includes characterization of semiconductor components with emphasis on ultrafast spectroscopy of semiconductor optical amplifiers.

Jesper Mørk received the M.Sc. and Ph.D. degrees from the Technical University of Denmark, Lyngby, Denmark, in 1986 and 1988, respectively.

Since 2002, he has been a Professor in semiconductor devices for optical communication systems and is responsible for modeling and theory in the Nanophotonics competence area at the Department for Communications, Optics and Materials (COM), Technical University of Denmark. His current research interests are in the area of device physics, in particular ultrafast devices for optical signal processing, quantum-dot structures, and noise in nonlinear devices.

Jørn M. Hvam received the M.Sc. degree in electrical engineering and the Ph.D. degree in physics from the Technical University of Denmark, Lyngby, Denmark, in 1969 and 1971, respectively.

From 1971 to 1993, he held various positions at universities and research laboratories in Denmark, France, and the United States. Since 1995, he has been a Full Professor at the Technical University of Denmark where he currently heads the nanophotonics at the Communication, Optics and Materials (COM) Research Center. His scientific research concerns the optical and optoelectronic properties of semiconductors. Over the last decade he has been mainly engaged in the fabrication and optical characterization of low-dimensional semiconductors, with special emphasis on ultrafast spectroscopy and dynamics of semiconductor nanostructures and devices. He heads national research programs on Semiconductor Components for Optical Signal Processing (SCOOP) and NanoSystems Engineering (NANOS). He has authored and coauthored more than 275 scientific papers appearing in international journals and books. He has given numerous invited talks and lectures at international conferences, meetings, and summer schools. 
Ortwin Hess received the Dipl. Phys. and Dr.rer.nat degrees in physics at theTechnical University of Berlin, Berlin, Germany, in 1990 and 1993 , respectively.

He was a Research Associate at Heriot-Watt University, Edinburgh, U.K., and the University of Tucson, Tuscon, AZ, and was a Postdoctoral Researcher at University of Marburg, Marburg, Germany. From 1994 to 2003, he was Head of the Theoretical Quantum Electronics Group, Institute of Technical Physics, DLR Stuttgart, Stuttgart, Germany. He has a Habilitation in Theoretical Physics at the University of Stuttgart, Stuttgart, Germany (1997), and became Adjunct Professor in 1998. He was a Visiting Professor at Stanford University, Stanford, CA (1997-1998), and the University of Munich, Munich, Germany
(2000-2001). Since March 2003, he has held the Chair of Computational Quantum Electronics, Advanced Technology Institute. He is Head of the Theory and Advanced Computation Group, University of Surrey, Surrey, U.K. His research interests are focused on the theory and mathematical modeling of the ultrafast optics and complex laser dynamics (quantum-dot lasers, control of spatiotemporal laser dynamics, ultrafast effects in active semiconductor media), nanophotonic materials (quantum dots, photonic crystals, synthetic opals, liquid crystals, biomolecules), biomodeling, as well as nanothermodynamics and nanorheology. 